\title{
Tumoren zielgerichtet entfernen? Hochauflösender Ultraschall im Operationssaal macht's möglich
}

Leipzig - Brustkrebspatientinnen nach der ersten Operation einen zusätzlichen Eingriff ersparen?! Dank ultraschallunterstütztem Operieren wird das immer häufiger möglich, denn so kann der Operateur besonders exakt erkennen, wo er schneidet - und das erkrankte Gewebe sehr zielgerichtet entfernen. Laut einer aktuellen Studie [1] wird bei rund 1 Drittel der Patientinnen eine weitere Operation unnötig. In der Hirnchirurgie können dank des Ultraschalls Ausfallerscheinungen verhindert werden. Welche Voraussetzungen ein Operateur für So- nografie-gesteuertes Operieren braucht und wie außerdem mittels hochintensiven fokussierten Ultraschalls (HIFU) krankes Gewebe durch Wärme zielgenau zerstört werden kann, erklärten Experten der Deutschen Gesellschaft für Ultraschall in der Medizin e. V. (DEGUM) auf einer Pressekonferenz in Leipzig im Rahmen des Dreiländertreffens der deutschen, österreichischen und Schweizer Ultraschallfachgesellschaften.

Der Ultraschall in der Medizin wird immer häufiger auch im Operationssaal genutzt.
Der Operateur legt dabei eine steril bezogene Ultraschallsonde im offenen Körper auf das betroffene Organ und erkennt exakt, wo der Tumor liegt. Er schneidet damit präzise nur das Gewebe heraus, das entfernt werden muss. Die Sonografie wird also dafür genutzt, Operationsinstrumente ultraschallgesteuert zielgenau in Organe zu bringen, ohne angrenzende Gewebe zu zerstören. „Die heutige Lebertumorchirurgie zum Beispiel wäre ohne intraoperative Sonografie nicht mehr denkbar“, sagt Dr. med. Matthias Wüstner vom Zentrum für Radiologie, Neuroradiologie, Sonografie 
und Nuklearmedizin am Krankenhaus der Barmherzigen Brüder Trier und Leiter der DEGUM-Sektion Chirurgie. „Durch die Ultraschalluntersuchung wird das heutige Maß an Präzision und Vollständigkeit bei der Entfernung von bösartigen Lebertumoren ermöglicht."

Eine noch elementarere Bedeutung hat das ultraschallunterstützte Operieren bei Hirntumoren, da der Neurochirurg noch präziser arbeiten muss - denn wenn gesundes, funktionsfähiges Hirngewebe versehentlich bei einer Operation entfernt wird, sind die verlorenen Funktionen nur schwer oder gar nicht mehr wiederherzustellen. Die Folge können Ausfallerscheinungen unterschiedlicher Ausprägung sein.

Bei Brustkrebs konnte in einer aktuellen Studie [1] nachgewiesen werden, dass durch die ultraschallassistierte im Vergleich zur konventionellen Tumorresektion signifikant weniger Patientinnen nachoperiert werden mussten. „Ein Ultraschallgerät sollte Bestandteil jedes Brustoperationssaals sein “, fordert deshalb Professor Dr. med. Markus Hahn, Leiter der Experimentellen Senologie am Universitätsklinikum Tübingen, Mitglied im DEGUM-Vorstand und diesjähriger Kongresspräsident des Dreiländertreffens.

Wichtigste Voraussetzung für das Sonografie-gesteuerte Operieren sind speziell ausgebildete Ärzte, die kompetent mit Ultraschallverfahren umgehen können. Der interdisziplinäre Arbeitskreis für „Interventionellen Ultraschall“ der DEGUM hat ein 2-Modul-Konzept entwickelt, in dem Ärzte das ultraschallassistierte Operieren erlernen. „Die Schwierigkeit ist, dass man dabei mit 2 Händen 3-dimensional arbeiten muss“, sagt Dr. Wüstner. Mit einer Hand muss die Ultraschallsonde so geführt werden, dass die Anatomie abgebildet wird. Mit der zweiten Hand wird operiert.

Ultraschall kann in der modernen Medizin aber noch viel mehr als nur Bilder zu erzeugen. Wenn Ultraschallwellen gebündelt und mit hoher Intensität gezielt in den Körper eingeleitet werden, so erzeugen sie Wärme, mit der Gewebe punktgenau und umgebungsschonend zerstört werden kann. Insbesondere bei gut-, aber auch bösartigen Tumoren wird diese Technik (HIFU high intensity focused ultrasound) eingesetzt. Anwendungsgebiete finden sich zum Beispiel im Bereich von Prostatakrebs, nicht operablem Bauchspeicheldrüsenkrebs oder Fibroadenomen der Brustdrüse, die gutartig sind. „Der flächendeckende Einsatz des hochintensiven fokussierten Ultraschalls ist noch lange kein Standard“, sagt Professor Hahn. Die DEGUM fördert deshalb die wissenschaftliche Erforschung solcher „thermodestruktiver" Verfahren, um das Spektrum der minimalinvasiven, operationsersetzenden Verfahren immer weiter voranzubringen.

\section{Literatur}

[1] Hoffmann J et al. Ultraschallassistierte Tumorresektion beim Mammakarzinom eine prospektive, randomisierte, monozentrische Studie (MAC 001). Senologie 2019; 16: 203-209 